\title{
Distribuição de Anadenanthera colubrina var. cebil (Griseb.) Altschul em uma área
}

\author{
de caatinga \\ Distribution of Anadenanthera colubrina var. cebil (Griseb.) Altschul in a caatinga área \\ Distribución de Anadenanthera colubrina var. cebil (Griseb.) Altschul en una zona de caatinga
}

Recebido: 19/09/2021 | Revisado: 26/09/2021 | Aceito: 27/09/2021 | Publicado: 29/09/2021

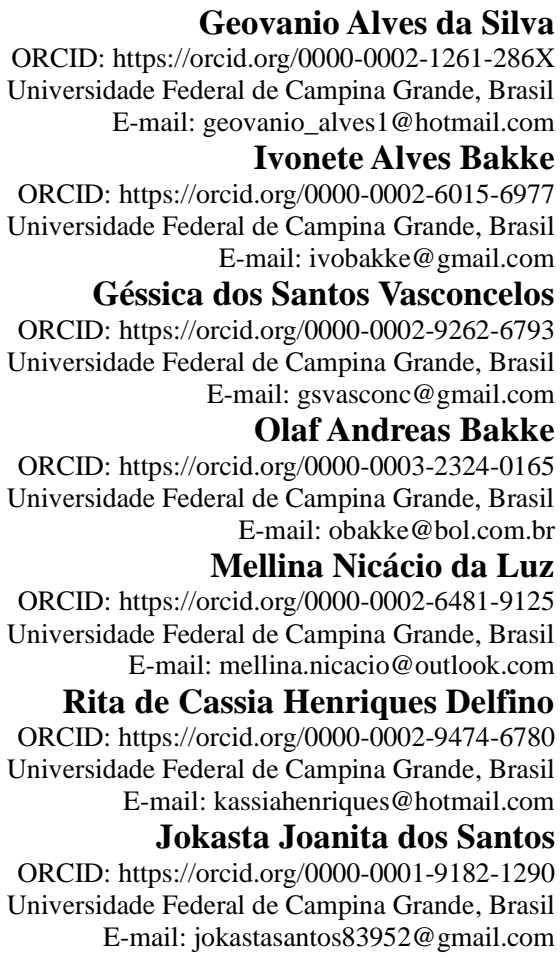

\section{Resumo}

Os processos de regeneração e de sucessão que ocorrem nas florestas nativas permitem a continuidade das espécies e fornecem informações acerca de sua estrutura e distribuição nas comunidades florestais. Estes processos estão intimamente relacionados ao grau de exploração da vegetação, aos fatores edafoclimáticos e também à preservação dos remanescentes florestais. Este trabalho teve como objetivo verificar a ocorrência dos indivíduos de A. colubrina e sua distribuição nas diferentes classes diamétricas em área de caatinga na Fazenda Lameirão município de Santa Terezinha-PB. O método utilizado para a contagem e medições de altura e diâmetro dos indivíduos desta espécie $A$. colubrina foi o caminhamento. Durante o percurso, todos os indivíduos de A. colubrina encontrados no trajeto foram mensurados a circunferência à altura do peito $(\mathrm{CAP}) \leq 6 \mathrm{~cm}$, e altura total, utilizando uma fita métrica $(\mathrm{cm})$ e régua graduada (GR 500 professional) $(\mathrm{cm})$, respectivamente. Os valores obtidos das circunferências foram transformados em diâmetros $(\mathrm{CAP} / \pi)$, e a partir destes, obteve-se a diferença entre esses valores e estabelecido o intervalo entre as classes diamétricas. Foram inventariados 99 indivíduos, cuja maioria apresentou altura superior a $5 \mathrm{~m}$ e DAP médio 14,33 cm. De acordo com os valores de DAP obtidos, os exemplares foram distribuídos em cinco classes diamétricas com amplitude de $5,73 \mathrm{~cm}$ em cada classe. A maior quantidade de indivíduos se concentrou nas classes II e III. A $A$. colubrina ocorre de forma aglomerada. A maior quantidade de indivíduos nas três primeiras classes diamétricas, indica um alto recrutamento de indivíduos juvenis nessas classes.

Palavras-chave: Aglomeração; Classes diamétricas; Sucessão secundária.

\begin{abstract}
The regeneration and succession processes that occur in native forests allow the continuity of species and provide information about their structure and distribution in forest communities. These processes are closely related to the degree of vegetation exploitation, edaphoclimatic factors and also to the preservation of forest remnants. This work aimed to verify the occurrence of individuals of A. colubrina and its distribution in the different diametric classes in the caatinga area at Fazenda Lameirão, municipality of Santa Terezinha-PB. The method used for counting and
\end{abstract}


measuring height and diameter of individuals of this species A. colubrina was walking. During the route, all individuals of A. colubrina found in the route were measured circumference at breast height (CAP) $<6 \mathrm{~cm}$, and total height, using a tape measure $(\mathrm{cm})$ and graduated ruler (GR 500 professional) $(\mathrm{cm})$, respectively. The values obtained from the circumferences were transformed into diameters (CAP / $\pi$ ), and from these, the difference between these values was obtained and the interval between the diametric classes was established. 99 individuals were inventoried, most of which had a height greater than $5 \mathrm{~m}$ and an average DBH $14.33 \mathrm{~cm}$. According to the DAP values obtained, the specimens were distributed in five diametric classes with an amplitude of $5.73 \mathrm{~cm}$ in each class. The largest number of individuals was concentrated in classes II and III. A. colubrina occurs in an agglomerated form. The greater number of individuals in the first three diametric classes, indicates a high recruitment of juvenile individuals in these classes.

Keywords: Agglomeration; Diametric classes; Secondary succession.

\section{Resumen}

Los procesos de regeneración y sucesión que ocurren en los bosques nativos permiten la continuidad de las especies y brindan información sobre su estructura y distribución en las comunidades forestales. Estos procesos están estrechamente relacionados con el grado de explotación de la vegetación, los factores edafoclimáticos y también con la preservación de los remanentes forestales. Este trabajo tuvo como objetivo verificar la ocurrencia de individuos de A. colubrina y su distribución en las diferentes clases diamétricas en el área de caatinga en Fazenda Lameirão, municipio de Santa Terezinha-PB. El método utilizado para contar y medir la altura y el diámetro de los individuos de esta especie A. colubrina fue caminar. Durante el recorrido, a todos los individuos de A. colubrina encontrados en el recorrido se les midió la circunferencia a la altura del pecho (CAP) $<6 \mathrm{~cm}$, y la altura total, utilizando una cinta métrica (cm) y regla graduada (GR 500 profesional) $(\mathrm{cm})$, respectivamente. Los valores obtenidos de las circunferencias se transformaron en diámetros (CAP $/ \pi$ ), y a partir de estos se obtuvo la diferencia entre estos valores y se estableció el intervalo entre las clases diamétricas. Se inventariaron 99 individuos, la mayoría de los cuales tenían una altura superior a 5 my un DAP promedio de 14,33 cm. De acuerdo con los valores de DAP obtenidos, los ejemplares se distribuyeron en cinco clases diamétricas con una amplitud de $5,73 \mathrm{~cm}$ en cada clase. El mayor número de individuos se concentró en las clases II y III. A. colubrina se presenta en forma aglomerada. El mayor número de individuos en las tres primeras clases diamétricas, indica un alto reclutamiento de individuos juveniles en estas clases.

Palabras clave: Aglomeración; Clases diamétricas; Sucesión secundaria.

\section{Introdução}

A exploração desordenada do bioma Caatinga, através das práticas antrópicas insustentáveis, desencadeia um intenso processo de desequilíbrio ambiental provocado notadamente pelo desmatamento e uso inadequado do solo. De um modo geral, a exploração da vegetação nativa ocorre sem seguir os critérios que assegurem a sua continuidade nos ecossistemas e a própria segurança do homem do campo. A presença de determinada espécie em uma área e seu potencial de comercialização e de renda, são os motivadores para sua exploração, porém os aspectos responsáveis pela sua existência e seu papel na estrutura da floresta são pouco observados e considerados, tampouco, os efeitos de sua retirada no equilíbrio ambiental.

As espécies arbóreas encontram-se distribuídas em grupos ecológicos de acordo com suas exigências por luz, umidade, solo e interações bióticas, as quais oferecem informações que facilitam o manejo (Almeida, et al., 2015). Dentre as espécies arbóreas nativas da caatinga o angico (Anadenanthera colubrina var. cebil (Griseb.) Altschul), é uma planta heliófila, perenifólia a semicaducifólia, presente na sucessão secundária inicial, que ocorre desde ambientes secos a mais úmidos, onde pode atingir até $35 \mathrm{~m}$ de altura (Medeiros, et al., 2016).

Para tomada de decisão e estratégias que visem o manejo sustentável da Caatinga, pesquisas sobre a composição e estrutura das florestas nativas são necessários, dessa forma, identificar a maneira que as espécies se distribuem é pré-requisito para compreender o dinamismo das populações vegetais (Santana, et al. 2016).

De acordo com (Machado, et al. 2010), uma floresta pode ser avaliada através da distribuição dos indivíduos arbóreos em classes de diâmetro. Esta classificação possibilita o reconhecimento das espécies presentes, permite entender a dinâmica de uma floresta, deduzir sobre as formas de exploração no passado e planejar o seu modelo de exploração (Santana, et al., 2011). O conhecimento da flora e de sua estrutura é fundamental para a elaboração de planos de manejos sustentáveis que visem explorar as florestas e preservá-las para as gerações futuras (Bambolim, et al., 2018). 
Os estudos da estrutura de uma floresta são realizados considerando dois aspectos: a estrutura horizontal que possibilita uma análise dos parâmetros fitossociológicos como densidade, dominância, frequência e importância das espécies; enquanto a que a estrutura vertical permite avaliar o desenvolvimento e estágio de sucessão da floresta com base nos diferentes estratos arbóreos (Guedes \& Krupek, 2016).

Estudos sobre a ocorrência de espécies e de sua distribuição diamétrica em áreas nativas de caatinga fornecem informações elementares para tomadas de decisões na aplicação de técnicas de manejo florestal ou conservação destes (Nascimento, et al., 2001). Desse modo, considerando a presença de vários indivíduos de A. colubrina em área de caatinga remanescente em estado de sucessão secundário, este estudo visa responder o seguinte questionamento: Como ocorre a distribuição desta espécie em área de caatinga em estado preservado, com ausência de exploração acerca de 30 anos?

A ausência de informações sobre as espécies da caatinga aliada a práticas inadequadas de manejo e exploração desordenada, bem como a ineficiência das políticas de reflorestamento que vise à reposição das árvores exploradas e, principalmente, a falta de outras opções de matéria-prima está colocando em risco o esgotamento dessa espécie florestal (Diniz, et al., 2006). Assim, este trabalho teve como objetivo verificar a ocorrência dos indivíduos de A. colubrina e sua distribuição nas diferentes classes diamétricas.

\section{Metodologia}

\section{Localização e caracterização da área}

O estudo foi desenvolvido na Fazenda Lameirão pertencente à Universidade Federal de Campina Grande - CSTR, Campus Patos, localizada geograficamente nas coordenadas $7^{\circ} 02 ’ 56,8^{\prime}$ latitude Sul e 37²9’36,2" longitude Oeste, no município de Santa Terezinha - PB, a uma altitude de $300 \mathrm{~m}$ acima do nível do mar (Figura 1).

Figura 1. Localização da área estudada na fazenda Lameirão, Santa Terezinha, PB.

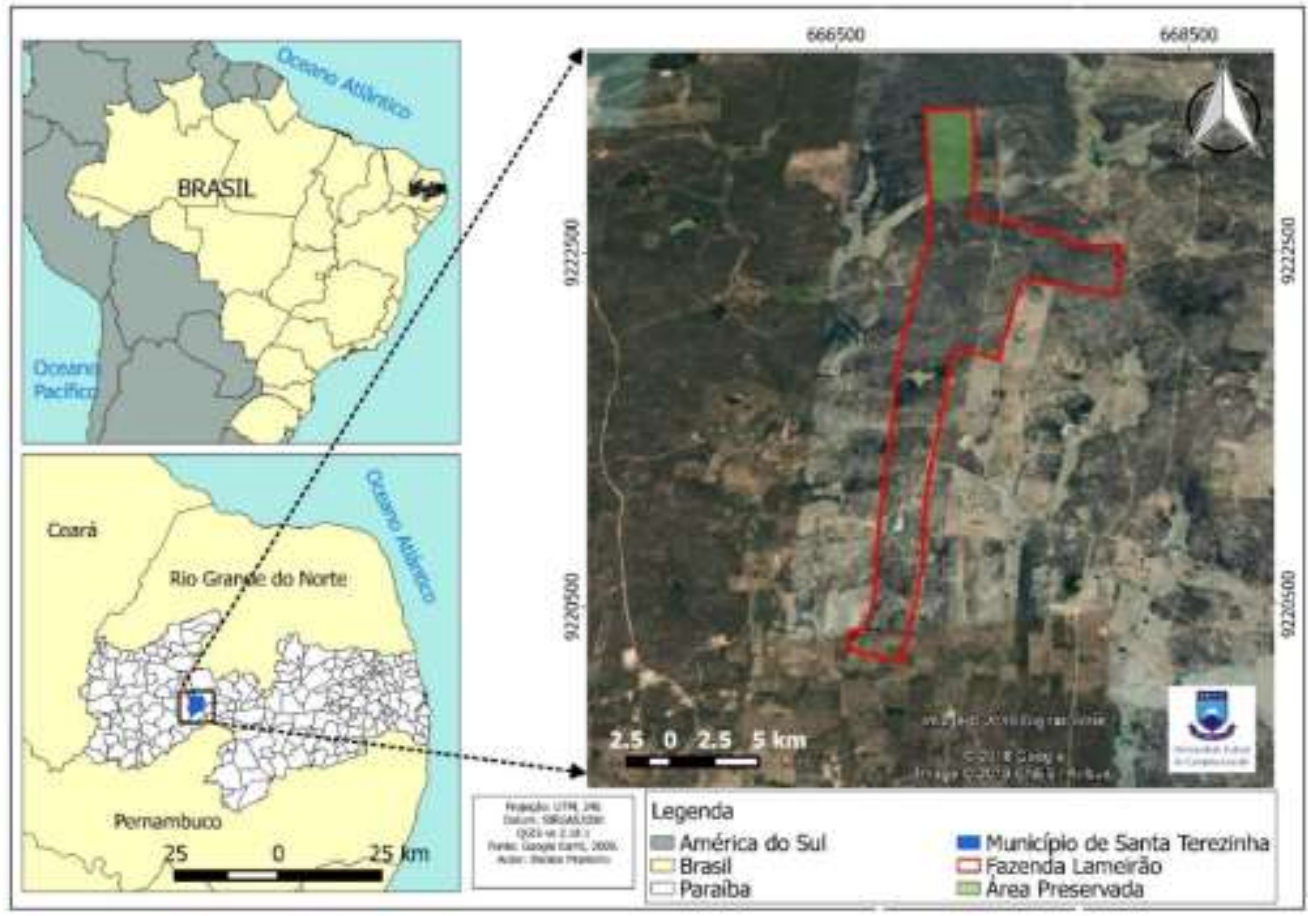

Fonte: Anjos (2018). 
De acordo com a classificação de (Alvares, et al. 2014), o clima da região é BShw' (quente e seco), caracterizado por estação chuvosa com variações na quantidade e na distribuição de precipitação mensal e anual, e outra seca. A média anual de precipitação dos últimos 27 anos foi de $839,1 \mathrm{~mm}$, a de temperatura foi de $25,2^{\circ} \mathrm{C}$, com a mínima registrada de $20,1^{\circ} \mathrm{C}$ e a máxima de $32,2^{\circ} \mathrm{C}$.

A área estudada tem 19 hectares, encontra-se em pousio, sem exploração madeireira por um período de aproximadamente 30 anos. Nestes dois últimos anos, seis bovinos pastejam na área durante a estação seca, especialmente nos meses de outubro a dezembro, quando há escassez de alimento para os animais.

É composta por uma vegetação arbóreo-arbustiva em processo de sucessão secundária, com a predominância de espécies pioneiras como: Mimosa tenuiflora, Piptadenia stipulaceae, Poicianella. pyramidalis e também espécies secundárias em menor quantidade tais como Myracrodruon urundeuva, Ambura cearensis, Handroanthus impetiginosus, Erythroxylum pungens, e muitos indivíduos de Anadenanthera colubrina.

\section{Procedimentos adotados para coleta dos dados}

Para Koche (2011), para alcançar o conhecimento científico, deve-se buscar métodos científicos. Neste sentido, Bacon (1979), afirma que o método científico deve seguir os seguintes passos: realizar experimentos sobre o problema investigado; formular hipóteses; repetir experimentos.

Sendo assim, afim de conhecer o comportamento da A. Colubrina em uma área de pousio, foi inicialmente realizado o reconhecimento e a medição da área de ocorrência da espécie. O método utilizado para a contagem e medições de altura e diâmetro dos indivíduos desta espécie A. colubrina foi o caminhamento adaptado de (Filgueiras, et al., 1994) em aproximadamente três hectares. A escolha deste método e da área (três hectares) foi devido ao comportamento de aglomeração verificado para a espécie na área, uma vez que esta ocorre de forma concentrada (agrupada) às proximidades de um riacho temporário que corta a área de floresta preservada.

Durante o percurso, todos os indivíduos de A. colubrina encontrados no trajeto foram mensurados a circunferência à altura do peito (CAP) $\leq 6 \mathrm{~cm}$, e altura total, utilizando uma fita métrica $(\mathrm{cm})$ e régua graduada (GR 500 professional) $(\mathrm{cm})$, respectivamente. O número de indivíduos coletado foi de acordo com a ocorrência dos exemplares durante o caminhamento.

\section{Distribuição das classes diâmetro}

Os valores obtidos das circunferências foram transformados em diâmetros $(\mathrm{CAP} / \pi)$, e a partir destes, obteve-se a diferença entre esses valores e estabelecido o intervalo entre as classes diamétricas, adaptado do Comitê Técnico Científico da Rede de Manejo Florestal da Caatinga (2005) (Quadro 1).

Quadro 1. Distribuição das classes diamétricas dos indivíduos de A. colubrina inventariados a Fazenda Lameirão Santa Terezinha-PB.

\begin{tabular}{|c|c|}
\hline Classes & Intervalos (cm) \\
\hline I & $3,50-9,23$ \\
\hline II & $9,24-14,96$ \\
\hline III & $14,97-20,69$ \\
\hline IV & $20,70-26,42$ \\
\hline V & $26,43-32,15$ \\
\hline
\end{tabular}

Fonte: Silva et al. (2018). 
Para construção dos histogramas das classes diamétricas e de altura foram utilizados os valores confeccionados no Excel, no qual foram separados em cinco classes.

\section{Resultados e Discussão}

Foram inventariados 99 indivíduos de A. colubrina cuja maioria (94) apresentou altura superior a 5 m e DAP médio $14,33 \mathrm{~cm}$ na população amostrada. De acordo com os valores de DAP obtidos, os exemplares foram distribuídos em cinco classes diamétricas com amplitude de $5,73 \mathrm{~cm}$ em cada classe. A maior quantidade de indivíduos se concentrou nas classes II com 34 indivíduos e III com 32. Nas classes I, IV e V foram registrados 22, 9 e 2 exemplares, respectivamente. Na Figura 2 visualiza-se o percentual de indivíduos (A) e os respectivos valores médios de DAP nas classes diamétricas (B).

Figura 2. Percentual de indivíduos de A. colubrina presentes nos intervalos das classes (A) e médias dos valores (B) dos diâmetros nas respectivas classes na fazenda Lameirão, Santa Terezinha, PB.
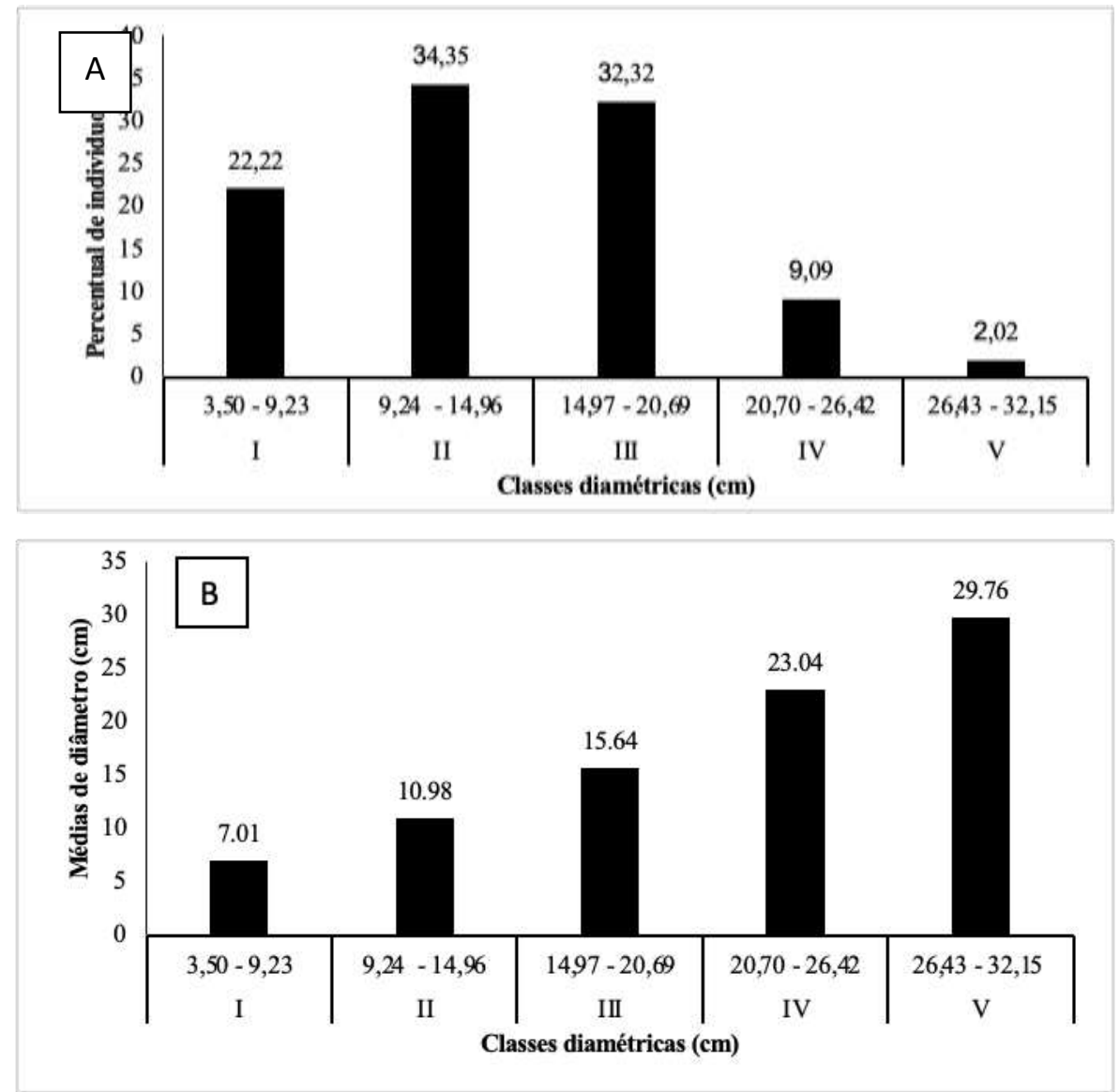

Fonte: Silva et al. (2018).

Analisando as Figuras 2 A e B, verifica-se uma maior quantidade de indivíduos nas três primeiras classes diamétricas, I (22 indivíduos), II (34) e III (32), com médias de diâmetros relativamente baixos, indicando um alto recrutamento de indivíduos juvenis nessas classes. Este comportamento pode estar associado ao período com ausência de intervenção antrópica (cerca de 30 anos), o que favorece o processo natural de sucessão e estabelecimento da espécie.

Para manter o equilíbrio da população da A. colubrina é necessário que se tenha indivíduos nas classes de tamanho inferiores, uma vez que estes representam a regeneração da espécie e a evolução para as classes subsequentes. Os resultados destes estudos corroboram ao desenvolvido por (Carlixto Júnior, et al., 2011), ao analisarem a distribuição diamétrica da $M$. 
tenuiflora em dois fragmentos de caatinga - PE, sendo um submetido ao corte raso há cerca de 40 anos e outro em estado de preservação por um período superior a este. Os autores constataram que a distribuição diamétrica da M. tenuiflora na área mais preservada apresentou um maior número de indivíduos nas primeiras classes de diâmetro e redução gradativa nas demais.

A distribuição espacial dos indivíduos de A. colubrina na área confirmou sua tendência de ocorrer de forma agrupada. De acordo com (Tierschnabel, et al., 2018) alguns fatores bióticos e abióticos são determinantes para a agregação dos indivíduos de uma espécie, dentre eles destacam-se a forma de dispersão barocórica (pela ação da gravidade), que favorece o acúmulo de sementes na serapilheira abaixo da copa das árvores adultas.

A agregação de espécies também foi observada em estudos de caatinga - PE por (Maragon et al., 2013), e enfatizam que tal fato, decorre das relações inter e intraespecíficas e seus diferentes níveis de funcionamento, assim como fatores ambientais, como solos rasos e perturbações naturais no ambiente.

No presente estudo, enfatiza que os indivíduos desta espécie encontravam-se restritos às proximidades do riacho temporário, onde também se beneficiavam da umidade do solo promovida pela infiltração da água durante o período chuvoso, fazendo parte da composição ripária daquele ambiente.

No que se refere aos indivíduos com $\mathrm{CAP} \leq 6,0 \mathrm{~cm}$, considerados regenerantes, não foram observados durante o caminhamento. Este resultado pode ser explicado por duas razões: a primeira pela dificuldade em identificar os exemplares na estação seca, quando as plantas se encontravam desprovidas de folhas, dificultando dessa forma o reconhecimento da espécie. A segunda possibilidade, provavelmente, a menos aceitável, seria a ausência destes indivíduos na área.

(Santana et al., 2011) afirmam que em florestas xerófilas e preservadas, é comum a mortalidade de indivíduos regenerantes devido aos fatores já mencionados acima, os quais variam entre as espécies, o nível de preservação e as condições ambientais, porém sua existência ou ausência irá determinar a continuidade do componente florestal. Além destes fatores, deve-se considerar o período entre o processo germinativo e o estabelecimento do indivíduo, o qual é caracterizado como o mais susceptível aos ataques dos herbívoros e alta mortalidade em função dos aspectos climáticos.

(Medeiros et al., 2016), de um certo modo, explicam os resultados do presente estudo ao verificarem a abundância de indivíduos regenerantes de A. colubrina em um fragmento de brejo de altitude em Bananeiras, PB seguida de alta mortalidade. Este comportamento permite inferir que apesar da dispersão barocórica, da grande quantidade de sementes desta espécie e de sua rápida germinação após as condições favoráveis, é necessário que os demais fatores estejam em equilíbrio para que as plântulas ultrapassem as dificuldades inerentes à esta fase e alcancem o estabelecimento nos estádios posteriores.

Desse modo, a ausência de dados de regeneração natural desta espécie nesta área, deve ser analisada com cautela e repetir o censo durante a estação chuvosa, a fim de assegurar qualquer uma das duas possibilidades mencionadas acima e permitir inferências mais concretas acerca do comportamento desta espécie na área estudada.

É importante frisar a relação direta entre as florestas e distribuição das chuvas, bem como o desenvolvimento de comunidades florestais em áreas sob deficiência hídrica, especialmente a adaptação das espécies a esta condição e sua continuidade no ecossistema (Martins, 2012b).

Para o município de Santa Terezinha - PB, foi possível verificar que a precipitação total que ocorreu na área durante o ano de 2018, até o mês de novembro do corrente ano supracitado, equivale a 691,95 $\mathrm{mm}$ distribuídos entre os meses de janeiro a maio, com maior concentração em fevereiro, março e abril (Figura 3). 
Figura 3. Precipitação registrada no município de Santa Teresinha, PB, no período estudado.

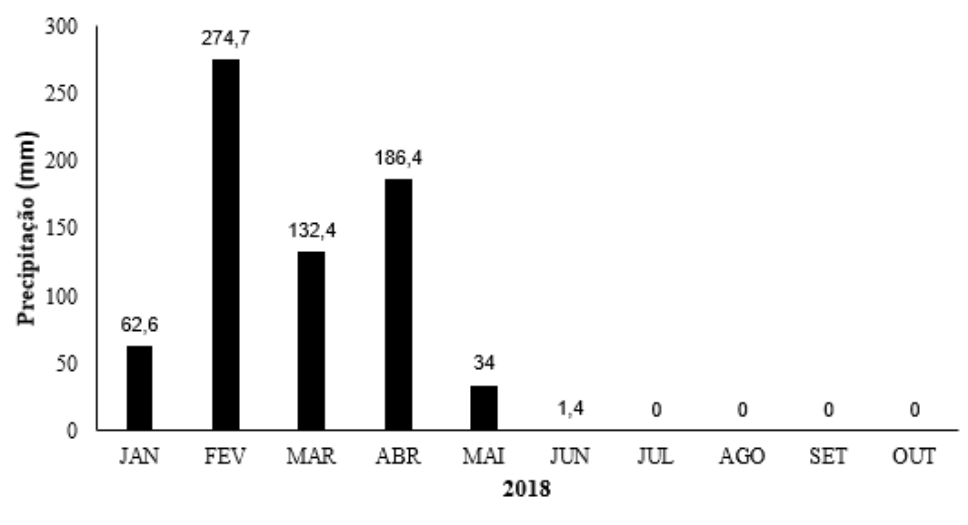

Fonte: AESA (2018).

Este comportamento é inerente à região semiárida, onde se constata que a maioria das chuvas ocorre nos primeiros meses do ano, e o restante dos meses fica submetido aos altos índices de insolação e, muitas vezes, aos elevados déficits hídricos. Limeira (2008) ressalta que a má distribuição das chuvas e/ou a escassez ao longo do ano, comprometem o desenvolvimento da vegetação nativa, as relações de equilíbrio dos ecossistemas, a umidade do solo, o armazenamento de água e as atividades agrícolas, das quais depende o homem do campo.

No presente estudo, verificou-se que a espécie A. colubrina ocorre de forma agrupada nas proximidades de um leito de um riacho temporário que corta a área de fragmento florestal em preservação. Este comportamento corrobora as afirmativas de (Medeiros, et al., 2016), ao verificarem a ocorrência desta espécie em matas ciliares, confirmando a sua relevância na manutenção e equilíbrio desses ecossistemas.

Outro elemento a ser considerado para entender os processos e as relações existentes em uma comunidade florestal é o solo e seus atributos físicos e de fertilidade. Na tabela 1 encontra-se o resultado da análise química da área estudada.

Tabela 1. Analise química do solo de área de caatinga preservada na fazenda Lameirão, Santa Teresina, PB.

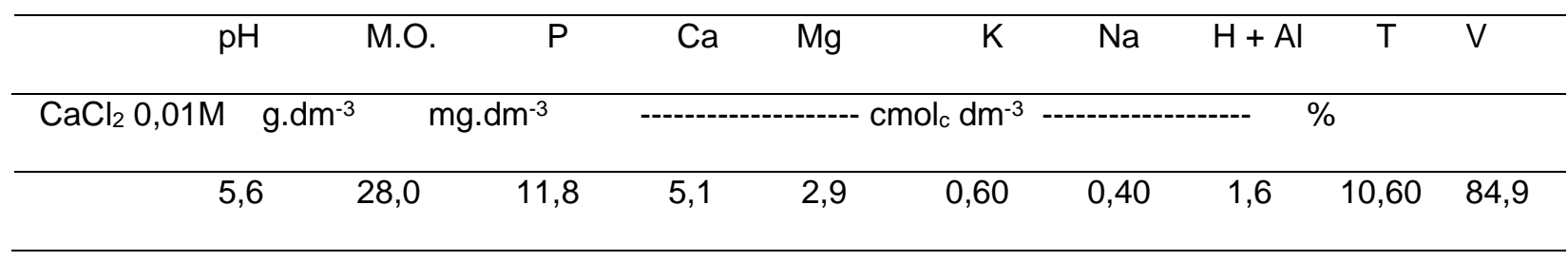

Fonte: Silva et al. (2018).

O solo da área foi caracterizado como franco arenoso (USDA - Departamento de Agricultura dos Estados Unidos) e de acordo com a tabela acima, tem $\mathrm{pH}(5,6)$ considerado levemente ácido, que não compromete o desenvolvimento dos vegetais especialmente por se tratar de uma área com vegetal nativa. A soma de bases, $(10,60)$ e a saturação de bases $(84,9 \%)$, caracterizam um solo eutrófico, jovem e revelam os benefícios da ausência da ação antrópica durante os 30 anos.

O resultado pode ser explicado pela baixa densidade da vegetação e as características edafoclimáticas da região, notadamente, a irregularidade das chuvas, as altas temperaturas e os atributos inerentes à formação e intemperismo do solo e a reciclagem de nutrientes. 


\section{Conclusão}

A Anadanthera colubrina ocorre de forma aglomerada na área de caatinga preservada da Fazenda Lameirão, preferencialmente ao longo do riacho temporário.

A maior quantidade de indivíduos nas três primeiras classes diamétricas, indica um alto recrutamento de indivíduos juvenis nessas classes.

Devido à ausência da ação antrópica na área estudada é possível observar o avanço da sucessão secundária com espécies características deste grupo ecológico, como a A. colubrina.

É necessário acompanhar a dinâmica de regeneração da A. colubrina na área estudada durante todo o ano, especialmente nas duas estações seca e chuvosa.

\section{Referências}

Almeida, C. M.; Araújo, M. M.; Longhi, S. J., Rovedder, A. P., Sccoti, M. S. V., Aimi, M. A. S. C., Tonetto, T. S. (2015). Análise de agrupamentos em remanescente de Floresta Estacional Decidual. Ciência Florestal, Santa Maria, 25 (3), 781-789. https://doi.org/10.5902/1980509819682

Alvares, C. A.; Stape, J. L.; Sentelhas, P. C.; Gonçalves, J. L. M.; Gerd Sparovek, G. (2014). Köppen's climate clasification map for Brazil. Meteorologische Zeit. Stuttgart, Alemanha, 22 (6), 711-728.

Bacon, F. (1979,) Novum Organum. 2. ed. São Paulo: Abril Cultural, p. 1-231 (Coleção Os Pensadores, Victor Civita);

Bambolim, A., Donde, A. R., Wojciechowsk, J. C. (2018). Análise fitossociológica e estrutura florística de uma floresta estacional decidual. Revista de Agricultura Neotropical, Cassilândia-MS, 5 (2), 62-68.

Calixto Júnior, J. T.; Drumond, M. A. Alves Junior, F. T. (2011). Estrutura e distribuição espacial de mimosa tenuiflora (Willd.) Poir. em dois fragmentos de Caatinga em Pernambuco. Revista Caatinga, Mossoró, 24 (2), 95-100.

Chazdon, R. (2012). Regeneração de florestas tropicais. Boletim do Museu Paraense Emílio Goeldi. Ciências Naturais. 7 (3), p. 195-218.

Diniz, C. E. F.; Paes, J. B.; Marinho, I. V.; Lima, C. R. (2006). Avaliação do potencial tanífero de seis espécies florestais de ocorrência no semi-árido brasileiro. Cerne, Lavras, 12 (3), 232-238.

Filgueiras, T.; Brochado, A. L.; Nogueira, P. E.; Guala, G. F. (1994). Caminhamentos: um método expedito para levantamentos florísticos qualitativos. Cadernos de Geociências. (12), 39-44.

Guedes, J.; Krupek, R. A. (2016). Florística e fitossociologia do componente arbóreo de um fragmento de floresta ombrófila densa do estado de São Paulo. Revista Acta Biológica Catarinense. Joinville, SC, 3 (1), 12-24.

Köche, J. C. (2011). Fundamentos de metodologia científica: teoria da ciência e iniciação à pesquisa. Petrópolis, RJ: Vozes.

Lacerda, G. L. B. et al., (2019). Análise temporal da vegetação na Sub-Bacia Do Rio Do Peixe, Paraíba/Brasil. Brazilian Journal of Development., Curitiba, 5 (8), 11794-11807. DOI:10.34117/bjdv5 n8 - 043

Machado, E. L. M.; Gonzaga, A. P. D; Carvalho, W. A. C; Souza, J. S.; Higuchi, P.; Santos R. M.; Silva, A. C.; Oliveira Filho, A. T. (2010). Flutuações temporais nos padrões de distribuição diamétrica da comunidade arbóreo-arbustivo e de 15 populações em um fragmento florestal. Revista Árvore, Viçosa, 34 (4), 723-732. https://doi.org/10.1590/S0100-67622010000400017

Maragon, G. P.; Ferreira, R. L.; Silva, J. A. A.; Lira, D. F. S; Silva, E, A, Loureiro, G. H. (2013). Estrutura e padrão espacial da vegetação em uma área de Caatinga. FLORESTA, Curitiba, PR, 43 (1), 83 - 92. http://doi.org/10.5380/rf.v43i1.27807

Medeiros, R. L. S.; Souza, V. C.; Neto, M. A. B; Araújo, L.; Barbosa, A. S. (2016). Estrutura da regeneração natural de Anadenanthera colubrina em fragmento de brejo de altitude em Bananeiras, PB. Pesquisa Florestal Brasileira. doi: 10.4336/2016.pfb.36.86.887

Nascimento, A. R. T.; Longhi, S. J.; Brena, D. A. (2001). Estrutura e padrões de distribuição espacial de espécies arbóreas em uma amostra de floresta ombrófila mista em Nova Prata, RS. Ciência Florestal, Santa Maria, 11 (1), 105-119. http://doi.org/10.5902/19805098

Santana, J. A. S.; Santana Júnior, J. A. S.; Barreto, W. S.; Ferreira, A. T. S. (2016). Estrutura e distribuição espacial da vegetação da Caatinga na Estação Ecológica do Seridó, RN. Pesquisa Florestal Brasileira. Colombo, 36 (82), 355-361. https://doi.org/10.4336/2016.pfb.36.88.1002

Santana, J. A. S.; Vieira, F. A.; Pacheco, M. V.; Oliveira, P. R. S. (2011). Padrão de distribuição e estrutura diamétrica de Caesalpinia pyramidalis Tul. (Catingueira) na Caatinga do Seridó. Revista de Biologia e Ciências da Terra. 11 (1).

Santos, J. H. S.; Ferreira, R. L.; Silva, J. A. A.; Souza, A. L.; Santos, E. S.; Meunier, I. M. J. (2004). Distinção de grupos ecológicos de espécies florestais por meio de técnicas multivariadas. Revista Árvore, Viçosa-MG, 28 (3),387-396.

Tierschnabel, B. R.; Melo Júnior, J. C. F.; Dornelles, S. S. (2018). Dispersão de sementes e densidade populacional de Psychotria nuda (Rubiceae) em fragmento urbano de Floresta Ombróila Densa de Terras Baixas no sul do Brasil. Revista Biotemas, 31 (2), 9-18. 